\title{
Cytomixis and meiotic abnormalities during microsporogenesis are responsible for male sterility and chromosome variations in Houttuynia cordata
}

\author{
J.-Z. Guan, J.-J. Wang, Z.-H. Cheng, Y. Liu and Z.-Y. Li \\ Institute of Medicinal Plants, College of Plant Science and Technology, \\ Huazhong Agricultural University, Wuhan, P.R. China \\ Corresponding author: Z.-Y. Li / Y. Liu \\ E-mail: lizaiyun@mail.hzau.edu.cn / yanl@mail.hzau.edu.cn
}

Genet. Mol. Res. 11 (1): 121-130 (2012)

Received May 5, 2011

Accepted November 29, 2011

Published January 17, 2012

DOI http://dx.doi.org/10.4238/2012.January.17.2

\begin{abstract}
Houttuynia cordata (Saururaceae) is a leaf vegetable and a medicinal herb througout much of Asia. Cytomixis and meiotic abnormalities during microsporogenesis were found in two populations of $H$. cordata with different ploidy levels $(2 \mathrm{n}=38,96)$. Cytomixis occurred in pollen mother cells during meiosis at high frequencies and with variable degrees of chromatin/chromosome transfer. Meiotic abnormalities, such as chromosome laggards, asymmetric segregation and polyads, also prevailed in pollen mother cells at metaphase of the first division and later stages. They were caused by cytomixis and resulted in very low pollen viability and male sterility. Pollen mother cells from the population with $2 \mathrm{n}=38$ showed only simultaneous cytokinesis, but most pollen mother cells from the population with $2 \mathrm{n}=96$ showed successive cytokinesis; a minority underwent simultaneous cytokinesis. Cytomixis and irregular meiotic divisions appear to be the origin of
\end{abstract}


the intraspecific polyploidy in this species, which has large variations in chromosome numbers.

Key words: Houttuynia cordata; Cytomixis; Microsporogenesis; Meiosis; Polyploidy

\section{INTRODUCTION}

Cytomixis refers to the phenomenon of chromatin/chromosome transfer between the proximate meiocytes through cytoplasmic channels or intercellular bridges. After it was first recorded by Kornicke (1901) in Crocus sativus, the phenomenon has been reported during microsporogenesis in a wide range of flowering plants (Wu et al., 2003; Ghaffari, 2006; Lattoo et al., 2006; Sheidai, 2008; Singhal and Kumar, 2008a,b; Maity and Datta, 2009). Besides in these natural species, cytomixis is also observed in artificially synthesized interspecific/generic hybrids (Li et al., 2005, 2009). The occurrence of cytomictic channels and chromatin transfer is found to be more prevalent in genetically, physiologically and biochemically imbalanced plants such as triploids, haploids, hybrids, apomicts, and aneuploids (Haroun, 1995; Nirmala and Rao, 1996), and also more prevalent among polyploids (Singhal et al., 2007) than their diploid counterparts. The results from many kinds of plants reveal that cytomixis is directly responsible for abnormal meiotic behavior, pollen grains of different sizes and pollen sterility, such as in Coix (Sapre and Deshpande, 1987), Alopecurus arundinaceus (Koul, 1990), Polygonum tomentosum (Haroun, 1995), Hordeum vulgare (Haroun, 1996), Brassica napus var. oleifera and B. campestris var. oleifera (Souza and Pagliarini, 1997), Vicia faba (Haroun et al., 2004), and Meconopsis aculeata (Singhal et al., 2008a). Cytomixis, which results in changes in gametic chromosome numbers through migration of chromosomes between adjacent meiocytes, could be considered as a process of evolutionary significance in plant populations (Falistocco et al., 1995; Morikawa and Leggett, 1996; Malallah and Attia, 2003; Boldrini et al., 2006; Li et al., 2009), although there are still some controversies as regards its role in evolution.

Houttuynia cordata Thunb., which is only the species in the Houttuynia genus and belongs to the family Saururaceae, is a perennial herb propagated by underground stems. This plant is mainly distributed in the Sino-Japanese regions of eastern Asia, ranging from Japan to the Himalayas through the Ryukyu Islands, Taiwan, and China, to Southeast Asia (Oginuma et al., 2007). In Japan, the species propagates by formation and separation of underground stems and by parthenogenesis (Shibata and Miyake, 1908; Mihara, 1960), whereas sexual reproduction has not been reported. It is also distributed in most regions of China and shows morphological variations, being commonly used for both vegetable and medicinal purposes. Its extracts contain active compounds, such as houttuynin and flavonoids.

The $H$. cordata populations of different origins have variable chromosome numbers with various basic chromosome numbers proposed. For the populations in China, Liang (1991) first suggested that the basic chromosome number is $x=12(2 n=24,96)$. The populations from the Sichuan Province in southwest China present $2 \mathrm{n}=36-126$ (36, $54,72,80,81,82,83,84,86,88,90,126)$ with $2 \mathrm{n}=81$ having the highest rate and $\mathrm{x}=9$ 
been suggested (Wu et al., 2003). In those populations in Japan, Nepal, Taiwan, Thailand, and unknown locations, various chromosome numbers have been identified $(2 \mathrm{n}=24-128)$ (Oginuma et al., 2007), and the $\mathrm{x}=12$ is first proposed and $\mathrm{x}=8$ has recently been suggested (Oginuma et al., 2007).

In the present study, the variable chromosome numbers in wide ranges $(2 \mathrm{n}=36$ 108) were recorded in 17 samples from six provinces/regions. The processes of male meiosis and microsporogenesis were investigated in detail for two populations with quite different ploidy levels $(2 \mathrm{n}=38,96)$. All individuals show an abnormal meiotic course such as chromosome laggards, asymmetric segregation and polyads. Furthermore, the cytomixis occurs at various stages of meiosis with different frequencies. The relationships of the irregular meiotic divisions and cytomixis with the variable chromosome numbers, ploidy levels and male sterility are discussed.

\section{MATERIAL AND METHODS}

The 17 samples of wild growing $H$. cordata were collected from different regions in Hubei, Sichuan, Guizhou, Guangdong, Shenzhen, and Chongqing Provinces of China. They were planted in the medicinal plant garden on the campus of our University for study. They showed variable chromosome numbers $(2 \mathrm{n}=36-108)$. Two populations were characterized for their male meiotic divisions, one with $2 \mathrm{n}=96$ (Y01xsh, Figure 1a) from the Hubei Province in central China, another with $2 \mathrm{n}=38$ (Y09sch, Figure 1b) from the Sichuan Province in southwest China.

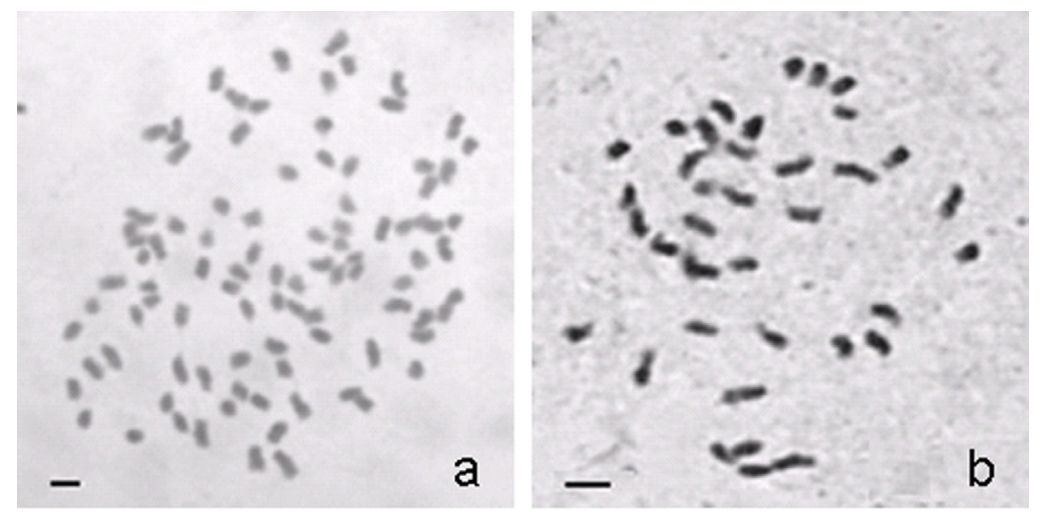

Figure 1. Different chromosome numbers in root-tip cells of Houttuynia cordata. $\mathbf{a} .2 \mathrm{n}=96 . \mathbf{b} .2 \mathrm{n}=38$. Bar $=2 \mu \mathrm{m}$.

Chromosome numbers were determined in root-tip cells and at least 50 cells were observed for each sample. Root tips of about $0.5 \mathrm{~cm}$ in length were pretreated with $1 \mathrm{mM} 8$-hydroxyquinoline for $6 \mathrm{~h}$ at $4^{\circ} \mathrm{C}$ and fixed in 3 ethanol: 1 acetic acid for at least $24 \mathrm{~h}$ and then transferred to $70 \%$ ethanol and stored at $4{ }^{\circ} \mathrm{C}$ until use. The spikelets at the ideal stages for meiotic studies and for pollen development were fixed directly and then stored at $4^{\circ} \mathrm{C}$ for use. Chromosome preparations followed the method of Li et al. (1995). Pollen viability was estimated as the percentage of pollen grains stained with $1 \%$ aceto-carmine. Preparations were checked and photomicrographs taken with the microscope Nikon Eclipse 80i (Japan) and a CCD camera. 


\section{RESULTS}

\section{Cytomixis in pollen mother cells (PMCs)}

Cytomixis involving transfer of chromatin material among proximate PMCs at various stages of meiosis (Figures 2a-h, 3a-h) was observed in all individuals of the two populations with $2 \mathrm{n}=38$ (Y09sch) (Figure 1b), 2n = 96 (Y01xsh) (Figure 1a). The migration of chromatin occurred through one or more chromatin strands. These chromatin strands usually had a broader head and an end that tapered towards the donor PMC, which was more obvious at prophase I. Frequently, only 2-3 PMCs at the same stages were involved in cytomixis but more were also occasionally included (Figure 3a). Simultaneous transfer of chromatin from one PMC to two other proximate PMCs also occurred (Figure 3a). Different amounts of chromatin were transferred between or among PMCs in each event (Figures 2a-h, 3a-e), from a chromatin mass to probably single chromosome. Therefore, PMCs at diakinesis from the same plant were observed to have quite different numbers of bi-/univalents (Figure 4a,b). The transferred chromatin did not fuse with the main chromosome complement, but formed extra chromatin masses, which lagged behind for the division progression (Figure 2b,c,e). The chromatin transfer also occurred in tetrads (Figure 3f-h), even at high frequency $(28.44 \%)$ in population Y01xsh. The transfer direction in tetrads was not easily recognizable, as the microspores were enclosed by a wall of callose.

The frequencies of cytomixis in PMCs at every stage were comparable in the two populations, being higher at prophase I. The data for the second meiotic division and microsporogenesis were not given for the limited numbers of PMCs observed (Table 1).

\section{Meiotic abnormalities}

PMCs at diakinesis without cytomixis in Y09sch $(2 n=38)$ showed the normal chromosome pairing as 19 bivalents (Figure 5a). The chromosomes in PMCs of Y01xsh $(2 n=96)$ were mainly paired as bivalents besides some univalents and multivalents, but precise pairings were difficult to determine for the high chromosome numbers (Figure $4 \mathrm{~b}$ ), and the variations in the numbers of bivalents likely resulted from the chromatin transfer (Figure 4a,b). Chromosome laggards and bridges prevailed in PMCs at metaphase I (MI) and later stages in the two populations, and more laggards appeared in PMCs from Y01xsh (Figures 4c-f, 5b-e). The percentages of PMCs with laggards during first and second divisions were much higher in Y01xsh than Y09sch (Table 1). As the consequence of chromosomal laggards, tetrads and micronuclei with different chromatin contents were produced in Y01xsh and Y09sch (Figure 4g,h). In the same anther, dyads (9.94\%), triads $(29.74 \%)$, tetrads $(22.19 \%)$, and polyads $(9.67 \%)$ were formed (Figure $4 \mathrm{~h}$, Table 1). Then, pollen grains with great size differences were produced in Y01xsh and the pollen stainability was only $0.38 \%$, for almost all pollen grains were shrunken or empty (Figure 4i). Y09sch produced microspores at eccentric uninucleate and binucleus stages with $82.3 \%$ stainable pollen grains (Figure $5 f$ ).

For the cytokinesis, PMCs from Y09sch only showed the simultaneous type. The majority of PMCs from Y01xsh showed successive cytokinesis, but the minority underwent simultaneous cytokinesis (Figure 6a-d). 


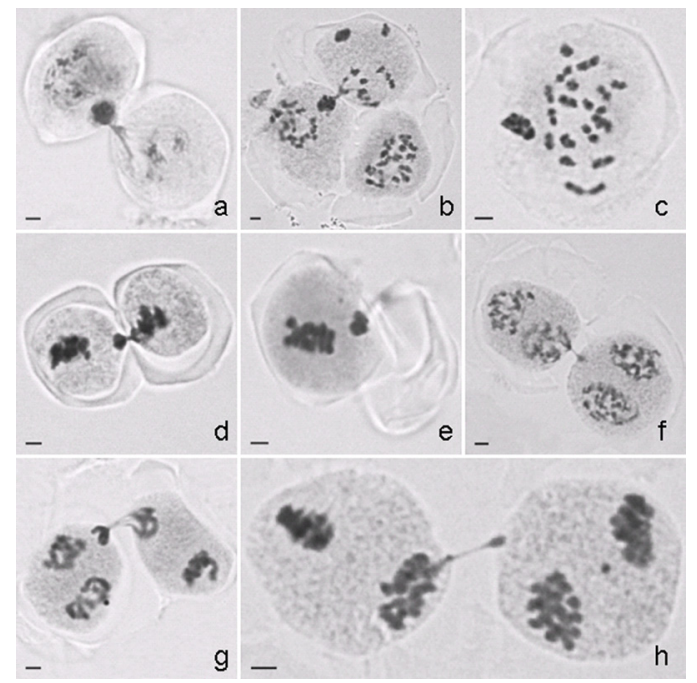

Figure 2. Cytomixis in Y09sch. a. Transfer of chromatin from the donor PMC leading to the formation of chromatin mass in recipient PMC at early prophase I. b. Chromatin transfer through one chromatin strand between two PMCs at diakinesis. The donor PMC has fewer bivalents than the recipient PMC and the one not involved in transfer. The chromatin transferred appears as one mass and has not yet formed bivalents. The donor PMC also has one chromatin mass, which was probably transferred from the other cell. c. One diakinesis PMC with 19 bivalents and one extra chromatin mass. d. Migration of chromosome at MI. e. One MI PMC with one extra chromatin mass. f. Transfer of chromatin at AI. g. Transfer of chromatin at TI. h. Transfer of chromatin at MII. Bar $=2 \mu \mathrm{m}$.

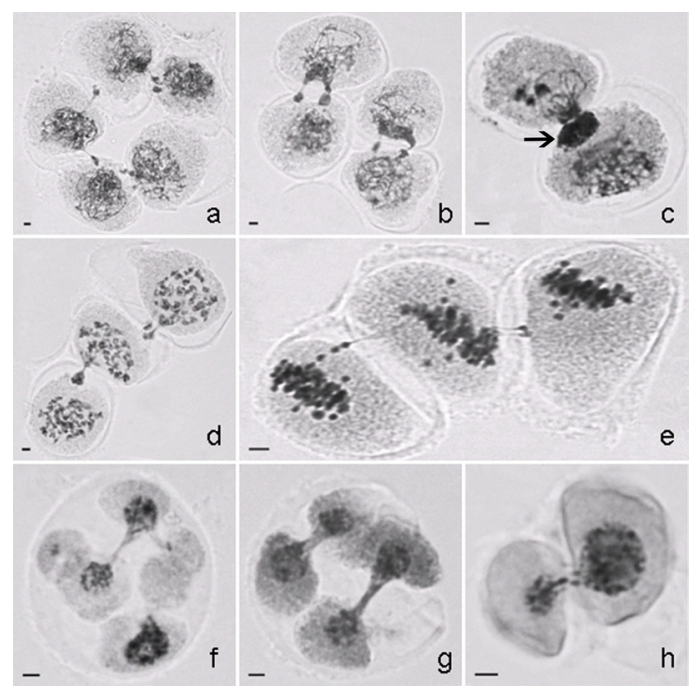

Figure 3. Cytomixis in Y01xsh. a. Transfer of chromatin among five PMCs at early prophase I. The cell at left has transferred its chromatin to two cells simultaneously. b. Chromatin transfer between two pairs of PMCs at early prophase I through two chromatin strands. c. Chromatin transfer of great extent from donor cell to recipient cell at early prophase I with the formation of a large chromatin mass (arrow). d. Chromatin transfer among three PMCs at diakinesis. e. Simultaneous transfer of chromatin from one cell (middle) to other two at MI through one (left side) or two (right side) chromatin strands. f. Hexads of different sizes with chromatin strands connecting three. $g$. Tetrads with large chromatin strands between two pairs. h. Two microspores with chromatin strands. Bar $=2 \mu \mathrm{m}$. 


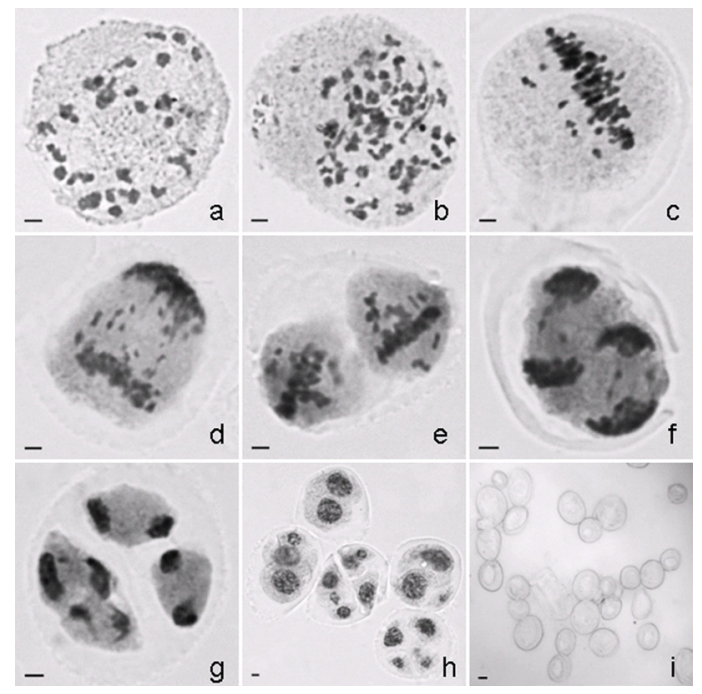

Figure 4. Abnormal meiosis in Y01xsh. Two diakinesis PMCs with fewer (a) or more bivalents (b). PMCs with laggards at MI (c), AI (d), MII (e), and AII (f). g. One tetrad, with two nuclei in each daughter cell from one extra division. h. Dyad, triad, tetrad, polyad with nuclei of different sizes. i. Unstainable pollen grains. Bar $=2 \mu \mathrm{m}$.

Table 1. The frequency of cytomixis and abnormal meiosis of two Houttuynia cordata populations.

\begin{tabular}{|c|c|c|c|c|}
\hline \multirow[t]{2}{*}{ Meiotic stages } & \multicolumn{2}{|c|}{ Cytomixis } & \multicolumn{2}{|c|}{ Meiotic abnormalities } \\
\hline & Y01 & Y09 & Y01 & Y09 \\
\hline Prophase I & $26.7 / 1128^{*}$ & $41.2 / 388$ & - & - \\
\hline Diakinesis & $19.6 / 566$ & $22.8 / 615$ & $-98.3 / 5376$ & - \\
\hline Metaphase I & $14.2 / 1086$ & $10.4 / 182$ & - & $12.4 / 1479$ \\
\hline Ana-/telophase I & - & $11.5 / 52$ & $35.6 / 838$ & $6.0 / 911$ \\
\hline Metaphase II & - & - & $96.8 / 416$ & $14.5 / 1051$ \\
\hline Ana-/telophase & - & - & $53.6 / 183$ & $3.7 / 322$ \\
\hline Tetrads & $28.4 / 966$ & - & - & - \\
\hline
\end{tabular}

*For PMCs at each division stage, the percentage and total number observed are given. - = data not obtained.

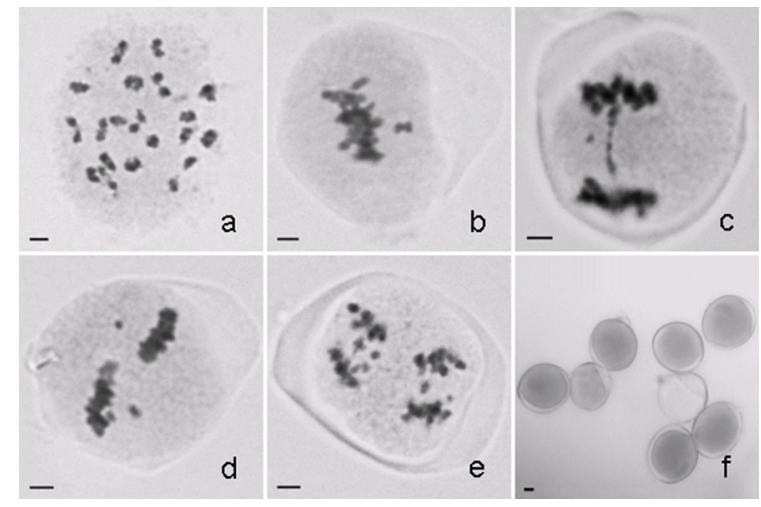

Figure 5. Abnormal meiosis in Y09sch. a. One diakinesis PMC with 19 bivalents. b. One MI PMC with laggards. c. One AI PMC with laggards and chromosomal bridge. d. One MII PMC with laggards. e. One AII PMC with laggards. f. Stainable and unstainable pollen grains. $\mathrm{Bar}=2 \mu \mathrm{m}$. 


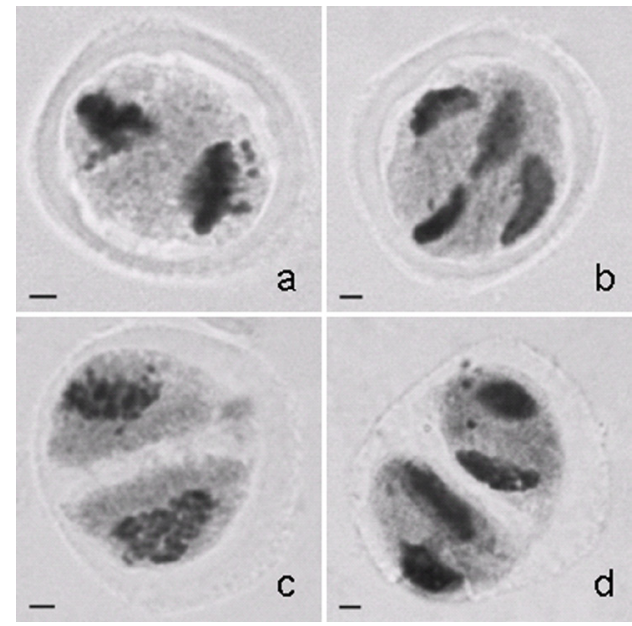

Figure 6. Two patterns of cytokinesis in PMCs of Y01xsh. Simultaneous cytokinesis at MII (a) and TII (b). Successive cytokinesis at MII (c) and TII (d).

\section{DISCUSSION}

The phenomenon of cytomixis and meiotic abnormalities were observed to occur at high frequencies in the two $H$. cordata populations with quite different chromosome numbers $(2 \mathrm{n}=38,96)$ collected in two distantly located provinces of China (Table 1, Figures 2a-h, 3a-h, 4a-i, 5a-f). The chromatin transfer occurred in PMCs from early prophase I to microspore stage. The cytomixis during microsporogenesis was also observed in PMCs at dyads and tetrads, and between microspores in some $H$. cordata populations from China (Wu et al., 2003). The present result that all individuals of these two populations showed cytomixis, together with the other of Wu et al. (2003), suggested that such phenomenon was characteristic of this species. The cytological processes of the chromatin transfer observed here was very similar to those in wild populations of Himalayan poppy (Meconopsis aculeata Royle) (Singhal and Kumar, 2008a). The transfer of chromatin material during microsporogenesis caused various meiosis abnormalities, reduced pollen viability and heterogeneous-sized pollen grains in the poppy.

The quite low pollen stainability and male sterility of the two $H$. cordata populations were probably attributable to the chromatin transfer. The extra chromatin masses formed by the transferred chromatin were usually located away from the main chromosome complement, delayed in division timing (Figures $2 \mathrm{~b}-\mathrm{e}, 3 \mathrm{~d}$ ), which was responsible for the chromosomal laggards, the micronuclei and the pollen grains of different sizes, and low viability or even sterility. The transfer of different amounts of chromatin resulted in the unbalanced complements of the PMCs involved and then microspores and pollen grains of again low viability or even sterility. On the contrary, no significant correlation was found between cytomixis and pollen viability in PMCs of Medicago sativa L., even if the cytomictic plants showed low values of pollen viability (Bellucci et al., 2003). The cytomixis was observed almost exclusively at the prophase of first meiotic division, and it was explained that the other meiotic abnormalities in first and second meiotic divisions, besides cytomixis, might have adverse effects on pollen viability. In Pinellia ternate with 
low seed-set and asexual reproduction through tubers and bulbils, the cytomixis occurred only during first division with low frequency, but abnormal karyokinesis and cytokinesis appeared in about 50\% of PMCs (Liu et al., 2012). Inversely, the cytomictic plants of diploid Dactylis had high pollen viability, as the cytomixis was one of the origins of $2 \mathrm{n}$ gametes (Falistocco et al., 1995). Therefore, the relationships between cytomixis and pollen viability varied depending on the species studied, which showed different cytological processes.

The $H$. cordata populations from different regions showed wide variations of chromosome numbers $(2 \mathrm{n}=24-128)$ and then intraspecific polyploidy, but its basic chromosome number is controversial, with $\mathrm{x}=8,9,12$ proposed for the samples studied (Oginuma et al., 2007). These cytotypes possibly originated from the cytomixis reported for the species (present study, Wu et al., 2003), because it has been shown to be a potential means to conserve the genetic heterozygosity of gametes (Veilleux, 1985) and additional means of phylogenetic evolution of karyotypes by reducing or increasing the basic series (Cheng et al., 1980, 1987), creation of aneuploids and polyploids (Sarvella, 1958; Falistocco et al., 1995). The aneuploidy male gametes with low viability or sterility were expected to have less contribution to spontaneous variations of chromosome numbers in $H$. cordata populations. The low pollen viability of this species is compensated by its perennial behavior, by the vegetative propagation through formation and separation of underground stems and by parthenogenesis (Shibata and Miyake, 1908; Mihara, 1960). The reproductive characteristics of parthenogenesis and microspore degeneration are widely accepted (e.g., Mabberley, 1998). The fact that the plants of these two populations observed here produced lots of viable seeds also suggested that they reproduced by parthenogenesis and were apomict. So it is necessary in future to observe the occurrence of cytomixis during the megasporogenesis and parthenogenesis of these populations. If cytomixis occurs in the female side, the absence of competition would mean that these aneuploidy gametes would function and produce aneuploid genotypes (Omara, 1976).

In angiosperms, generally two basic types of microsporogenesis are recognized: successive and simultaneous. Simultaneous microsporogenesis is plesiomorphic in angiosperms, although the successive type occurs in some early branching lineages and is especially characteristic of some monocotyledons (Furness and Rudall, 1999). The eudicot clade of angiosperms is characterized by simultaneous microsporogenesis and tricolpate pollen apertures. The occurrence of both simultaneous and successive types of microsporogenesis in Houttuynia populations also provides more clues for its special phylogenetic positions. It was originally classified into dicotyledons (Cantino et al., 2007), but was recently classified into the order Piperales and the clade magnoliids by APG II (The Angiosperm Phylogeny Group, 2003), while the magnoliids included those plants that did not belong to the monocots and eudicots. It would be of interest to study the types of microsporogenesis and pollen morphology in Houttuynia populations from more regions and the relationships with phylogenetics.

\section{REFERENCES}

Bellucci M, Roscini C and Mariani A (2003). Cytomixis in pollen mother cells of Medicago sativa L. J. Hered. 94: 512-516. Boldrini KR, Pagliarini MS and Valle CB (2006). Cell fusion and cytomixis during microsporogenesis in Brachiaria humidicola (Poaceae). South Afr. J. Bot. 72: 478-481. 
Cantino PD, Doyle JA, Graham SW, Judd WS, et al. (2007). Towards a phylogenetic nomenclature of Tracheophyta. Taxon 56: 822-846.

Cheng KC, Nie XW, Wang YX and Yang QL (1980). The relation between cytomixis and variation of chromosome numbers in pollen mother cells of rye (Secale cereals L.). Acta Bot. Sin. 22: 216-220.

Cheng KC, Quiglan Y and Yongsen Z (1987). The relationship between cytomixis, chromosome mutation and karyotype evolution in Lily. Caryologia 40: 243-259.

Falistocco E, Tosti N and Falcinelli M (1995). Cytomixis in pollen mother cells of diploid Dactylis, one of the origins of 2n gametes. J. Hered. 86: 448-453.

Furness CA and Rudall PJ (1999). Microsporogenesis in monocotyledons. Ann. Bot. 84: 475-499.

Ghaffari GM (2006). Occurrence of diploid and polyploidy microspores in Sorghum bicolor (Poaceae) is the result of cytomixis. Afr. J. Biotechnol. 5: 1450-1453.

Haroun SA (1995). Cytomixis in pollen mother cells of Polygonum tomentosum Schrank. Cytologia 60: 257-260.

Haroun SA (1996). Induced cytomixis and male sterility in pollen mother cells of Hordeum vulgare L. Delta J. Sci. 20: 172-183.

Haroun SA, Al Shehri AM and Al Wadie HM (2004). Cytomixis in the microsporogenesis of Vicia faba L. (Fabaceae). Cytologia 69: 7-11.

Kornicke M (1901). Über Ortsveränderung von Zellkarnern S B Niederhein. Ges. Nat. Heilk. Bonn. 14-25.

Koul KK (1990). Cytomixis in pollen mother cells of Alopecurus arundinaceus Poir. Cytologia 55: 169-173.

Lattoo SK, Khan S, Bamotra S and Dhar AK (2006). Cytomixis impairs meiosis and influences reproductive success in Chlorophytum comosum (Thunb.) Jacq. - an additional strategy and possible implications. J. Biosci. 31: 629-637.

Li XF, Liu SB, Gao JR, Lu WH, et al. (2005). Abnormal pollen development of bread wheat - Leymus mollis partial amphiploid. Euphytica 144: 247-253.

Li XF, Song ZQ, Feng DS and Wang HG (2009). Cytomixis in Thinopyrum intermedium, Thinopyrum ponticum and its hybrids with wheat. Cereal Res. Commun. 37: 353-361.

Li Z, Liu HL and Luo P (1995). Production and cytogenetics of intergeneric hybrids between Brassica napus and Orychophragmus violaceus. Theor. Appl. Genet. 91: 131-136.

Liang HX (1991). Karyomorphology of Gymnotheca and phylogeny of four genera in Saururaceae. Acta Bot. Yunnan 13: 303-307.

Liu Y, Hui RK, Deng RN, Wang JJ, et al. (2012). Abnormal male meiosis causes pollen sterility in medicinal polyploidy plant Pinellia ternate. Genet. Mol. Res. 11: (in press).

Mabberley DJ (1998). The Plant Book. A Portable Dictionary of the Vascular Plants. 2nd edn. Cambridge University Press, Cambridge.

Maity S and Datta AK (2009). Meiosis in nine species of jute (Corchorus). Indian J. Sci. Tech. 2: 27-29.

Malallah GA and Attia TA (2003). Cytomixis and its possible evolutionary role in a Kuwaiti population of Diplotaxis harra (Brassicaceae). Bot. J. Linn. Soc. 143: 169-175.

Mihara T (1960). On the reduction division of Houttuynia cordata Thunb. Bot. Mag. Tokyo 73: 498.

Morikawa T and Leggett JM (1996). Cytological and morphological variations in wild populations of Avena canariensis from the Canary Islands. Genes Genet. Syst. 71: 15-21.

Nirmala A and Rao PN (1996). Genesis of chromosome numerical mosaicism in higher plants. Nucleus 39: 151-175.

Oginuma K, Sato H, Kono Y, Chen S, et al. (2007). Intraspecific polyploidy of Houttuynia cordata and evolution of chromosome number in the Saururaceae. Chromosome Bot. 2: 87-91.

Omara MK (1976). Cytomixis in Lolium perenne. Chromosoma 55: 267-271.

Sapre AB and Deshpande DS (1987). A change in chromosome number due to cytomixis in an interspecific hybrid of Coix L. Cytologia 52: 167-174.

Sarvella P (1958). Cytomixis and loss of chromosomes in meiotic and somatic cells of Gossypium. Cytologia 23: 14-24.

Sheidai M (2008). Cytogenetic distinctiveness of sixty-six tetraploid cotton (Gossypium hirsutum L.) cultivars based on meiotic data. Acta Bot. Croat. 67: 209-220.

Shibata K and Miyake H (1908). Ueber Parthenogenesis bei Houttuynia cordata. Bot. Mag. Tokyo 22: 141-144.

Singhal VK and Kumar P (2008a). Impact of cytomixis on meiosis, pollen viability and pollen size in wild populations of Himalayan poppy (Meconopsis aculeata Royle). J. Biosci. 33: 371-380.

Singhal VK and Kumar P (2008b). Cytomixis during microsporogenesis in the diploid and tetraploid cytotypes of Withania somnifera (L.) Dunal, 1852 (Solanaceae). Comp. Cytogenet. 2: 85-92.

Singhal VK, Gill BS and Dhaliwal RS (2007). Status of chromosomal diversity in the hardwood tree species of Punjab State. J. Cytol. Genet. 8: 67-83.

Souza AM and Pagliarini MS (1997). Cytomixis in Brassica napus var. oleifera and Brassica campestris var. oleifera (Brassicaceae). Cytologia 62: 25-29. 
The Angiosperm Phylogeny Group (APG II) (2003). An update of the Angiosperm Phylogeny Group classification for the orders and families of flowering plants: APG II. Bot. J. Linn. Soc. 141: 399-436.

Veilleux R (1985). Diploid and polyploid gametes in crop plants: mechanisms of formation and utilization in plant breeding. Plant Breed. Rev. 3: 253-288.

Wu W, Zheng YL, Yang RW, Chen L, et al. (2003). Variation of the chromosome number and cytomixis of Houttuynia cordata from China. J. Syst. Evol. 41: 245-257. 